AN. MED. INTERNA (Madrid) Vol. 18, N. $^{\circ} 9$, pp. 483-485, 200

\title{
Tuberculosis miliar, ganglionar, pancreática y costal. Presentación clínica y revisión bibliográfica
}

\author{
I. INCHAURRAGA ÁLVAREZ, A. HERREJÓN SILVESTRE, P. PLAZA VALÍA, \\ R. BLANQUER OLIVAS
}

Servicio de Neumología. Hospital Dr. Peset. Valencia

MILIARY, GANGLIONAR, PANCREATIC AND COSTAL TUBERCU LOSIS. CLINICAL PRESENTATION AND BIBLIOGRAPHIC REVIEW

\begin{abstract}
RESUMEN
Se presenta un paciente de 42 años que ingresa en nuestro hospital con fiebre, dolor abdominal y patrón radiológico miliar. Tenía un aumento de amilasemia y amilasuria y de la lipasa sérica. El mantoux era negativo. En el estudio de TAC había engrosamiento costal, lesiones hepáticas focales múltiples y adenopatías necrosadas en zona peripancreática. Las biopsias transbronquiales y la punción pancreática mostraron lesiones granulomatosas necrotizantes, cultivándose $M$. tuberculosis complex en el broncoaspirado. El tratamiento específico normalizó todas las alteraciones pulmonares, costales y pancreáticas.
\end{abstract}

PALABRAS CLAVE: Tuberculosis miliar. Tuberculosis costal. Tuberculosis pancreática. Linfadenopatía tuberculosa.

\begin{abstract}
A 42-year-old man was admitted to the hospital with fever, abdomi nal pain and miliary radiological pattern.The amylase and lipase in serum, and the amylase in urine were increased. The tuberculin skin test was negative. The thoracic and abdominal CT scan showed costal afectation, multiple focal hepatic lesions and large necrosing peripancreatic lymphadenopathies. The transbronchial biopsy and the pancreatic punc tion demostrated granulomatous necrotic lesions, and $\mathrm{M}$. tuberculosis growed in the bronchial washing culture. The specific treatment norma lized the pulmonary, costal and pancreatic lesions.
\end{abstract}

KEY WORDS: Miliary tuberculosis. Costal tuberculosis. Pancreatic tuberculosis. Lymphadenopathy tuberculous.

Inchaurraga Álvarez I, Herrejón Silvestre A, Plaza Valía P, Blanquer Olivas R. Tuberculosis miliar, ganglionar, pancreática y costal. Presen tación clínica y revisión bibliográfica. An Med Interna (Madrid) 2001; 18: 483-485.

\section{INTRODUCCIÓN}

En la última década ha habido un cambio sustancial en la incidencia de la tuberculosis (TB) en el mundo desarrollado. En los Estados Unidos la tendencia epidemiológica de la TB varió bruscamente desde 1985 con un incremento progresivo en su incidencia hasta 1992, atribuido a la epidemia de VIH, a la inmigración de zonas de alta prevalencia y a la falta de programas de prevención. Desde 1992 la incidencia de TB en EE.UU. comienza a declinar por mejor control de la enfermedad (1).

Aunque la presentación habitual de la TB es exclusivamente pulmonar, existen formas inusuales de extraordinaria gravedad como son la TB miliar y meníngea. La TB miliar representa el $0,6 \%$ de la TB activa y el $11 \%$ de la extrapulmonar (2), con una mortalidad que oscila entre el 25 y el $50 \%$ (3), existiendo problemas diagnósticos, por lo que en ocasiones es un hallazgo necrópsico (4). Por otro lado, aunque la forma adenopática es relativamente frecuente, la aparición de TB pancreática o costal es excepcional, con escasas referencias en la bibliografía.

Presentamos un raro caso de TB miliar con afectación pancreática, costal y adenopática que evolucionó favorablemente con el tratamiento tuberculostático.

\section{CASO APORTADO}

Varón de 42 años, natural de Senegal, ingresa por fiebre de inicio reciente y patrón miliar en la radiografía de tórax. No tenía hábitos tóxicos. La exploración física era anodina salvo disminución del murmullo vesicular en la base derecha y dolor abdominal difuso. Hemograma era normal. VSG $45 \mathrm{~mm} / \mathrm{h}$. Ácido úrico $9,2 \mathrm{mg} / \mathrm{dl}$, CPK 293 UI/L, amilasa 536 UI/L, lipasa 696 UI/L., LDH 1023 UI/L, GOT $61 \mathrm{UI} / \mathrm{L}, \mathrm{GPT} 44 \mathrm{UI} / \mathrm{L}, \gamma$-GT $254 \mathrm{UI} / \mathrm{L}$ y FFAA $455 \mathrm{UI} / \mathrm{L}$ Amilasuria: $2860 \mathrm{UI} / \mathrm{L}$. Gasometría arterial con $\mathrm{FiO}_{2}$ 0,21: $\mathrm{pH}$ 7,44, $\mathrm{PO}_{2} 73$, $\mathrm{PCO}_{2} 33$ y $\mathrm{SaO}_{2} 95 \%$. Mantoux negativo. Rx de tórax: patrón miliar e infiltrado en LID (Fig. 1). TAC torácica: lesión nodular en el LID de base pleural, de $3 \times 3 \mathrm{~cm}$. Engrosamiento costal a nivel del $4^{\circ}$ arco

Trabajo aceptado: 28 de enero de 2000

Correspondencia: I. Inchaurraga Álvarez. Avda. Gaspar Aguilar, 90. 46017 Valencia. 
anterior izquierdo. RMN: tumoración hiperintensa con vaso en su porción inferior que se extiende hasta el área prevertebral. TAC helicoidal con estudio angiográfico: masa quística en LID con una arteria que se origina en tronco celiaco y se dirige hacia la masa pulmonar. TAC abdominal: lesiones focales hepáticas múltiples, de tamaño inferior a $1 \mathrm{~cm}$ así como grandes adenopatías necrosadas en zona peripancreática (Fig. 2). En la ecografía abdominal: Agrandamiento difuso de la cabeza pancreática y múltiples imágenes hipoecoicas a nivel retroperitoneal, peripancreático, hilio hepático y en tronco celíaco. Fibrobroncoscopia con biopsias transbronquiales: infiltración granulomatosa necrotizante. Ecocardiograma: ligera hipertrofia septal con regurgitación mitral leve. El estudio microbiológico inicial fue negativo. La serología del VIH fue negativa. Se practica PAAF pancreática que muestra lesión granulomatosa. Se inicia tratamiento tuberculostático estándar con normalización clínica, analítica y radiológica. A los 60 días se aisla $M$. tuberculosis en el cultivo de Lowenstein del BAS. El paciente rechazó la exéresis de su secuestro pulmonar.
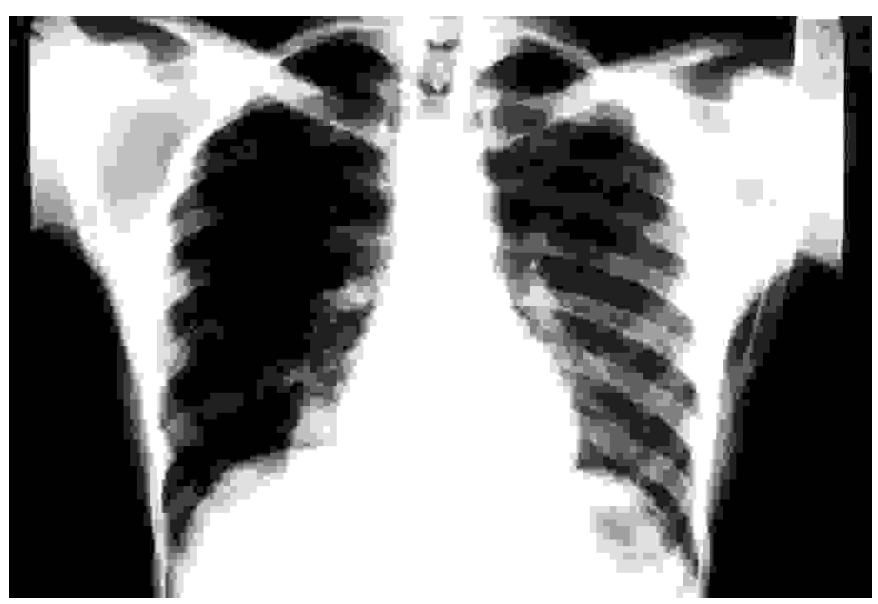

Fig. 1. Patrón miliar y masa paracardiaca derecha.

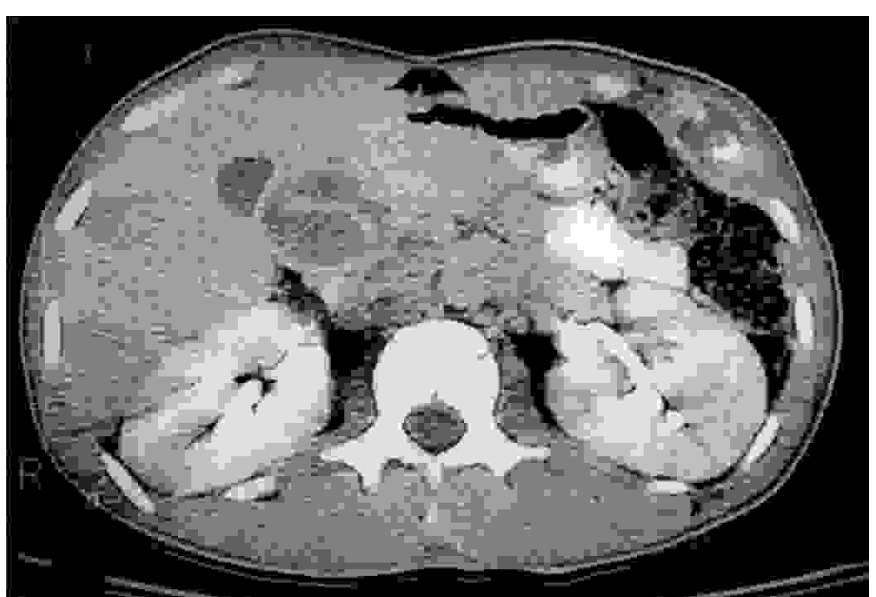

Fig. 2. Grandes adenopatías necróticas peripancreáticas.

\section{DISCUSIÓN}

La TB miliar es la diseminación hematógena de un foco establecido que produce numerosas lesiones simultáneas y del mismo tamaño que progresan a necrosis y caseificación. Predomina en negros (2:1) (2), la edad media es de 60 años y en dos tercios de los pacientes existen factores de riesgo como alcoholismo, diabetes, neoplasias o inmunodeficiencias (5). En el 59\% de las ocasiones el mantoux es positivo (2). Existen focos de TB activa en un $30 \%$ y la clínica habitual es de fiebre, síndrome constitucional y, con menor frecuencia, sintomatología digestiva.

En el $12 \%$ de los casos la Rx de tórax es normal, sobre todo en las dos primeras semanas del proceso (6). La Rx de tórax permite la identificación en el 59-69\% de las TB miliares (7), mostrando nódulos de menos de $3 \mathrm{~mm}$ (90\%). En etapas iniciales o casos dudosos, el TAC de alta resolución podría ser más sensible que la Rx de tórax $(8,9)$.

En el diagnóstico microbiológico se obtiene buen rendimiento con estudios repetidos de esputo y orina (hasta el 93\%) (10); en otras series, el diagnóstico en los esputos es del 76\%, $75 \%$ en aspirado gástrico, $59 \%$ en orina y $54 \%$ en el BAS (5). Las biopsias transbronquiales frecuentemente tienen una histología anormal, pero los cultivos son raramente positivos (5). En casos con esputos negativos, la broncoscopia con BAS, cepillado bronquial y biopsias transbronquiales diagnostica el $73 \%$ de los pacientes (11). Estos datos se confirman en otros estudios (12), pudiendo optar a otras técnicas diagnósticas con alto rendimiento, como las biopsias hepáticas y de médula ósea $(13,14)$. Nuestro diagnóstico se realizó con el cultivo del broncoaspirado.

Por otro lado, la incidencia de la TB extrapulmonar oscila del $11 \%$ del total $(15,16)$, al $25 \%$ (17), teniendo al parecer peor pronóstico que las formas pulmonares y de más difícil diagnóstico, con clínica insidiosa que puede demorar el diagnóstico hasta años (18). Así, la confirmación bacteriológica se consigue sólo en el $60 \%$. La ausencia de aislamiento microbiológico no excluye la tuberculosis, sobre todo en situaciones de alta sospecha clínica y prueba de tuberculina positiva (2).

Dentro de las formas extrapulmonares la más frecuente es la adenopática, siendo característica, aunque no exclusiva, la imagen tomográfica de baja atenuación del centro ganglionar, rodeado por un halo en anillo, con afectación mesentérica, peripancreática y retroperitoneal, dando raramente ictericia obstructiva (7). Nuestro caso mostró idénticas imágenes, con estudio histológico compatible con TB.

La afectación pancreática de la TB se da en el 4,7\% de la TB miliar, habiendo casos excepcionales de obstrucción biliar $(20,21)$ o pancreática y de pancreatitis aguda y crónica $(22,23)$. Se manifiesta principalmente por fiebre y dolor epigástrico, asociándose a TB miliar o inmunosupresión (24-27). Nuestro caso presentaba hiperamilasemia y aumento persistente de lipasa, sin ictericia, que se normalizaron con el tratamiento, siendo la punción-biopsia sugestiva de TB aunque con cultivos negativos. A veces, la TB pancreática se manifiesta como una masa pancreática que simula neoplasia, de difícil diagnóstico (28), pudiendo requerir laparotomía exploradora (29).

Por último, la TB costal es una rareza, mostrando la TAC de tórax destrucción ósea y cartilaginosa, con masas de tejido blando, posibles calcificaciones y patología pleuropulmonar subyacente. Se comporta de manera similar a la afectación de la pared torácica de la actinomicosis, siendo una manifestación infrecuente de la TB músculo-esquelética $(2 \%)$.

Sin embargo, los cartílagos costales y costillas son los lugares extrapulmonares más comunes de la TB en los 
ADVP, por diseminación hematógena o por contigüidad (30). Nuestro caso presentó dolor costal localizado y mostró una imagen radiológica compatible, aunque sin destrucción costal, que se resolvió con el tratamiento. En ocasiones esta destrucción costal puede aparentar una tumoración (31).

\section{Bibliografía}

1. McCray E, Weinbaunm CM, Braden CR, Onorato MI. The epidemiology of tuberculosis in the United States. Clins in Chest Med 1997; 18 (1): 99-113.

2. Álvarez S, McCabe WR. Extrapulmonary tuberculosis revisited: A review of experience at Boston city and other Hospitals. Medicine 1984; 63 (1): 43-44.

3. Chilvers SPJ, Leitch AG. Miliary tuberculosis in Edinburgh: a comparison between 1984-1992 and 1954-1967. Respir Med 1994; 88 (8): 609-611.

4. Ortiz J. Problemas actuales en el diagnóstico de la tuberculosis miliar. Rev Clín Esp 1985; 177 (9): 425-428.

5. Kim JH, Langston AA, Gallis HA. Miliary tuberculosis: epidemiology, clinical manifestations, diagnosis, and outcome. Rev Infect Dis 1990; 12 (4): 583-590.

6. Vidal R, Vilaplana M, Richart C, Morell F, Ruiz-Manzano J, Morera J. Presentación clínica y métodos diagnósticos en 113 casos de tuberculosis miliar. Med Clín (Barc) 1982; 78: 173-178.

7. Kwong JS, Carignan S, Kang EY, Müller NL, Fitzgerald JM. Miliary tuberculosis. Diagnostic accuracy of chest radiography. Chest 1996; 110: 339-342.

8. Oh YW, Kim YH, Lee NJ, Kim JH, Chung KB, Suh WH et al. Highresolution CT appearance of miliary tuberculosis. J Comput Assist Tomogr 1994; 18 (6): 862-866.

9. Optican RJ, Ost A, Ravin CE. High-resolution computed tomography in the diagnosis of miliary tuberculosis. Chest 1992; 102: 941-943.

10. Guerrero A, Erice A, Sanz-Hospital J, Gómez-Criado C, Gómez-Mampaso E. El diagnóstico bacteriológico de la tuberculosis miliar en la actualidad. Rev Clín Esp 1985; 177: 435-437.

11. Pant K, Chawla R, Mann PS, Jaggi OP. Fiberbronchoscopy in smearnegative miliary tuberculosis. Chest1989; 95 (5): 1151-1152.

12. Burk JR, Viroslav J, Bynum LJ. Miliary tuberculosis diagnosed by fiberoptic bronchoscopy and transbronchial biopsy. Tubercle 1978; 59 (2): 107-109.

13. Maartens G, Willcox PA, Benatar SR. Miliary tuberculosis: rapid diagnosis, hematologic abnormalities, and outcome in 109 treated adults. Am J Med 1990; 89 (3): 291-296.

14. Bodem ChR, Hamory BH, Taylor HM, Kleopfer L. Granulomatous bone marrow disease. A review of the literature and clinico-pathologic analysis of 58 cases. Medicine 1983; 62 (6): 372-377.

15. Farer LS, Lowell AM, Meador MP. Extrapulmonary tuberculosis in the United States. Am J Epidemiol 1979; 109: 205-217.
Concluimos con que la TB miliar es una patología grave, de difícil diagnóstico, que precisa habitualmente de la toma de muestras broncoscópicas y que puede manifestarse con afectación costal, adenopática y pancreática, cuya sospecha clínica requiere un tratamiento precoz a la espera de la confirmación microbiológica.

16. Menta JB, Dutt A, Harvill L, Mathews KM. Epidemiology of extrapulmonary tuberculosis. A comparative analysis with pre-AIDS era. Chest 1991; 99 (5): 1134-1138.

17. Steliamides S, Belmatong N, Fantin B. Manifestations and diagnosis of extrapulmonary tuberculosis. Rev Mal Respir 1997; 14 (5): S72-S87.

18. Elder NC. Extrapulmonary tuberculosis. A review. Arch Fam Med 1992; 1 (1): 91-98

19. Hulnick DH, Megibow AJ, Naidich DP, Hilton S, Cho KC, Balthazar EJ. Abdominal tuberculosis: CT evaluation. Radiology 1985; 157 : 199-204.

20. Crowson MC, Perry M, Burden E. Tuberculosis of the pancreas: a rare cause of obstructive jaundice. Br J Surg 1986; 73 (5): 373.

21. Ezratty A, Gumaste V, Rose E, Sachar DB, Tiscornia-Wasserman P. Pancreatic tuberculosis: a frecuently fatal but potentially curable disease. J Clin Gastroenterol 1990; 12 (1): 74-77.

22. Lundstedt C, Nyman R, Brismar J, Hugosson C, Kagevi I. Imaging of tuberculosis. II. Abdominal manifestations in 112 patients. Acta Radiol 1996; 37: 489-495

23. Stambler SB, Klibaner MI, Bliss ChM, Lamont JT. Tuberculous abscess of the pancreas. Gastroenterology 1982; 83: 922-925.

24. Boadas J, Rebasa P, Monill JM, Vallverdu H, Condomines J, Lluis F. Tuberculous pancreatic abcess: diferential diagnosis and favorable response to medical treatment. Gastroenterol Hepatol 1996; 19 (3): $156-158$.

25. Brusko G, Melvin WS, Fromkes JJ, Ellison EC. Pancreatic tuberculosis. Am Surg 1995; 61 (6): 513-515.

26. Pateron D, Naveau S, Brivet F, Bedossa P, Poynard T, Chaput JC. Acute tuberculous pancreatitis in a patient with acquired Inmunodeficiency syndrome. Gastroenterol Clin Biol 1990; 14 (1): 80-83.

27. Knowless KF, Saltman D, Robson HG, Lalonde R. Tuberculous pancreatitis. Tubercle 1990; 71 (1): 65-68.

28. Mourad FH, McLean A, Farthing MJ. Tuberculous pancreatitis: a diagnostic problem. Case report and review of literature. J Clin Gastroenterol 1995; 20 (3): 237-240.

29. Fischer G, Spengler U, Neubrand M, Sanerbruch T. Isolated tuberculosis of the pancreas masquerading as a pancreatic mass. Am J Gastroenterol 1995; 90 (12): 2227-2230.

30. Adler BD, Padley SPG, Müller NL. Tuberculosis of the chest wall; CT findings. J Comput Assist Tomogr 1993; 17 (2): 271-273.

31. Ip M, Chen NK, So SY, Chin SW, Lam WK. Unusual rib destruction in pleuropulmonary tuberculosis. Chest 1989; 85: 242-244. 